\title{
Unsupervised Clustering Separation in Cross-Layer Approach for UHF RFID Tag Collision Recovery
}

\author{
Yu Zeng, Haifeng $\mathrm{Wu}^{*}$ and Chuanxi Xing \\ School of Electrical and Information Technology, Yunnan University of Nationalities, 650500 Kunming, People's Republic of China \\ ${ }^{*}$ Corresponding author
}

\begin{abstract}
In passive ultra-high frequency (UHF) radio frequency identification (RFID) system, tag collision is generally resolved on a medium access control (MAC) layer. However, the collided signals are in fact recovered on a cross-physical (PHY)MAC layer to enhance identification efficiency. This paper adopts the cross-layer approach to propose an unsupervised clustering algorithm which could directly separate the collision RFID signal on the PHY layer. The advantage of the proposed algorithm is to have lower computational complexity than traditional unsupervised clustering methods, like k-means. Moreover, the proposed algorithm does not depend on prior known prefix signals as training data. Simulation results show that the proposed approach has better identification efficiency performance than traditional pure MAC-layer approaches regardless of the number of tags
\end{abstract}

Keywords- RFID; collision recovery; cross layer

\section{INTRODUCTION}

Passive radio-frequency identification (RFID) is the wireless non-contact use of radio-frequency electromagnetic fields to transfer data, for the purposes of automatically identifying and tracking tags attached to objects [1]. The passive RFID supports simultaneous multi-tag identification and hence offers higher identification efficiency than conventional bar code technology. In the passive system, an RFID reader identifies multiple tags on a shared wireless channel. Therefore, when two or more than two tags simultaneously transmit their signals to the reader, collision may happen and will disturb the identification. The multi-tag identification is actually a multi-access communication system. Collision resolution can be viewed as multi-user access. However, some well-known multi-access approaches, such as TDMA, FDMA, CDMA and OFDMA [2] might not be ideally fit for the passive RFID system due to the following reasons. First, the communication in the passive RFID system is a burst traffic model, where the number of unidentified tags might vary much. TDMA and FDMA are fixed resource allocation approaches and thus are not efficient for the burst traffic. Second, the passive RFID tags feature simple circuits for the low cost of the tags and thus are difficult to support some complex multi-access approaches, such as OFDMA. Finally, the passive tags operate on limited battery power which comes from a reader's continuous carrier transmission, whereas the CDMA approach requires bandwidth expansion and fast and efficient power control for each user.

In contrast to the multi-access approaches above, random multi-access approaches [3] are more appropriate for the passive RFID system. The approaches randomize the time of the tag identification. That is, the time when a tag responds is random. If two or more than two tags respond at the same time, they will collide with each other. In this case, the tags will reselect other time to respond until no collision happens. Owing to the time resource randomly allocated to the tags and its easy implementation, the approaches are very efficient for the burst traffic and widely applied to the passive RFID system.

However, the random multi-access approaches resolve the tag collision only on a medium access control (MAC) layer. Their identification efficiency is not high. The collided signals are in fact the superposition of the tag signals, which could be recovered directly on a physical (PHY) layer and thus enhance the identification efficiency [4-9]. In this paper, we will utilize the idea of the cross-layer approach and propose an unsupervised clustering algorithm which provides an approach to separate multiple UHF RFID tag collision on the PHY layer. The contribution of this paper is as follows.

First, the proposed unsupervised clustering separation has lower computational complexity. As we known, unsupervised algorithms, like k-means clustering method need to find the center of clusters via numerous iterations due to no training data[8]. And, their co mputational comple xity increases with the number of clusters. When the number of clusters increases dramatically, the complexity may be unavailable. On the other hand, our method uses a histogram to find a peak and be able to reduce the clustering complexity. Second, the performance of the proposed unsupervised clustering separation does not depend on a prefix signal. To reduce the complexity of the unsupervised clustering, a prior known prefix signal could be used for training date [5]. Due to the drift of the UHF prefix signal [15], however, the clustered centers sometimes are not correct. Owing to prefix signals being unused, the performance of our method is robust even under the condition of the drift.

\section{RELATED WORKS}

In a passive RFID system, multiple tag collision is generally resolved by random multi-access approaches on a MAC layer. The approaches can be categorized into tree-based and ALOHA-based algorithms [3]. Tree-based algorithms resolve the collision by muting subsets of tags that are involved in the collision [10]. Successively muting subsets finally leads to a tag's successful identification. A binary-tree algorith m [11] is widely applied in the tree-based algorithms due to its easy implementation. In the algorithm, the collided tags are divided into only two subsets. One is responding and the other is 
waiting. An international RFID standard, ISO 18000-6B [12] adopts the binary-tree algorithm to identify tags. However, the identification efficiency of the algorithm is not high, only 0.35 [11].

In ALOHA-based algorithms, an identification cycle will be divided into several time slots, and tags will select random slots to transmit signals to a reader $[13,14]$. If the tags are collided at a given slot, the tags will re-select another one until being identified. Dynamic frame length ALOHA is very popular in the ALOHA-based algorithms. Many RFID standards, such as ISO 18000-6A [12] and EPC C1 Gen 2 [15] both adopt the algorithm to identify tags. The algorithm not only divides an identification cycle into several slots but also forms several slots into a frame. Each tag responds randomly and only once in a frame. If a tag collides with others, it will enter the next frame to respond again. The advantage of the dynamic frame length ALOHA algorithm is to dynamically adjust each frame length to reduce collision or idle slots. The algorithm's identification efficiency is higher than the binarytree algorithm and arrives at a value of 0.37 [14].

Besides the MAC algorithms above, some hybrid MAC algorithms which mix tree-based and ALOHA-based algorithms are proposed in [16, 17]. They still divide an identification cycle into several slots which tags randomly select. If collision occurs in a slot, however, the collided tags in the slot will be split by trees at once and the other tags will wait until the splitting tree is finished. In contrast to the pure treebased or the pure ALOHA-based algorithms, the hybrid algorithms have a larger value of the identification efficiency, about 0.43 [16, 17].

In this paper, we adopt the cross-layer idea to propose a theoretic anti-collision framework on a cross-MAC-PHY layer. In contrast to the conventional pure MAC anti-collision algorithms, the proposed algorithm would have higher identification efficiency. Next, we will describe the MAC-PHY cross-layer approach.

\section{CROSS-LAYER APPROACH}

\section{A. Resolution on MAC}

Here, we will take the dynamic frame length ALOHA algorithm as an example of the tag identification on the MAClayer.

The dynamic frame length ALOHA is illustrated in Fig. 1(a). An identification cycle consists of several frames. For the first slot of the first frame, only one tag selects it. The slot thus is a readable slot. If no tags select a slot, such as the third slot in the first frame, the slot is an idle slot. Both of the second and the fourth slot in the first frame have two or more than two tags. These tags thus will be collided, and the collided tags will enter the next frame. In the dynamic frame length ALOHA algorithm, the frame length, i.e. the number of slots in the frame is dynamically adjusted according to the number of unidentified tags in the beginning of the frame. If we assume that the first frame length in Fig. 1(a) is equal to the number of tags, the second frame length will be adjusted to a smaller value because there are several tags which have already been identified. When all tags are identified, the identification cycle is finished. The last frame in Fig. 1(a) has no collision slots. This means that there are no unidentified tags. Therefore, the whole identification is completed.

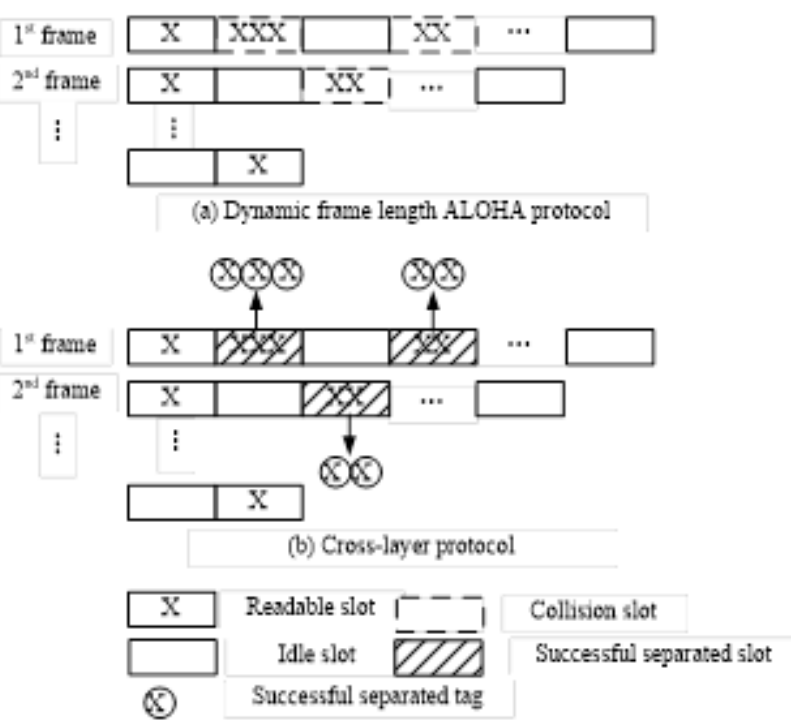

FIGURE I. A COMPARISON BETWEEN THEDYNAMIC FRAME LENGTH ALOHA ALGORITHM AND THE CROSS-LAYER APPROACH.

Now, we will firstly analyze the identification efficiency of the dynamic frame length ALOHA algorithm. Given one of the slots in a frame with a length $l, R$ tags allocated for the slot are a binomial distribution with $R$ Bernoulli experiments and $1 / l$ occupied probability. The probability of $r$ tags selecting the slot is therefore given by [6,13, 14 and 17]

$$
\rho_{r}=C_{R}^{r}(1 / l)^{r}(1-1 / l)^{R-r}
$$

And then, the expected number of the slots where $r$ tags respond simultaneously can be given by

$$
\alpha_{r}=l C_{R}^{r}(1 / l)^{r}(1-1 / l)^{R-r}
$$

From [3, 6, 14 and 16], the identification efficiency in the frame is a ratio between the number of readable slots and the number of total slots in the frame, i.e. $\eta=\alpha_{1} / l$. Thus, (2) will yield

$$
\eta=(1-1 / l)^{R-1} R / l
$$

The cross-layer approach is illustrated in Fig. 1(b), where tags still select random slots. Assume that the second and the fourth slot in the first frame still have two or more than two tags. Then both of the slots may not be regarded as collision slots any more. The collided tag signals in the slots can be separated on the PHY layer. If there are $r$ tags transmitting signals simultaneously in a slot, the expected number of tag signals which are successfully separated from the $r$ tag signals can be shown as 


$$
\bar{r}=\sum_{q=1}^{r} q \gamma_{r, q}
$$

where $\gamma_{r, q}$ denotes the probability that there are $q$ tag signals successfully separated from $r$ tag signals. From (1-4), the identification efficiency of the cross-layer approach can be given by

$$
\eta^{\prime}=\sum_{r=1}^{r_{\max }} \sum_{q=1}^{r} C_{R}^{r}(1 / l)^{r}(1-1 / l)^{R-r} q \gamma_{r, q}
$$

where $r_{\max }$ denotes the maximum number of tag signals from which some tag signals can be successfully separated. That is, no tag signals can be successfully separated when the number of collided tag signals is beyond $r_{\max }$. It could be proved that the efficiency $\eta^{\prime}$ in (5) will be greater than that in (3). That is, the identification performance of the cross-layer approach will be better than the pure dynamic frame length ALOHA.

\section{B. Recovery on $\mathrm{PHY}$}

Next, we describe how to separate some tags from collided tags on the PHY layer. We consider basic communication between several UHF tags and an RFID reader equipped with single receiving antenna. The tags use backscatter modulation to transmit signals to the reader. Given $N$ tags transmitting in a certain slot, each tag $n, n=0,1, \ldots N-1$ changes from absorbing energy into reflecting energy, by mis matching their antenna input impedance. After receiving the $N$ tag signals superposition, the reader will down convert the receive signals to the baseband. Hence, the complex-valued baseband signal at the receive antenna is $[3,4]$

$$
Z_{L}(t)=\sum_{n=0}^{N-1} h_{n} c_{n}(t)+L+n_{0}(t)
$$

where

$h_{n}=h_{n}^{f} h_{n}^{b} \sqrt{\Delta \sigma_{n}}$ is a flat fading linear time invariant channel in a very short time communication [4] in which $h_{n}^{f}$ and $h_{n}^{b}$ denote the forward channel (the reader to the $\operatorname{tag} n)$ and the backward channel (the $\operatorname{tag} n$ to the reader) coefficient, respectively and $\Delta \sigma_{n}$ is standardized coefficient of radar cross section.

$n_{0}(t)$ is the additive white Gaussian noise (AWGN) added at the reader.

$c_{n}(t)=\Sigma_{k=0}^{K-1} d_{n, k} g_{a_{n}}\left(t-k a_{n}-b_{n}\right)$ realizes an on-off keying that is complex values and unknown to the reader and features a different symbol period $a_{n}$ and a different symbol delay $b_{n}$ [3-5], $d_{n, k} \in\{0,1\}$ denotes the transmitted symbol and $g_{a_{n}}(t)$ denotes the pulse shape of the modulation signal.

A reader only discovers the carrier leakage $L$ during times where all tags are in their absorb state, i.e. $Z_{L}(t)=L$ when $d_{n, k}=0$. Such a period is defined in [5] before the tags respond. We can utilize this period to estimate the carrier leakage. Hence, we make

$$
Z(t)=Z_{L}(t)-L
$$

The problem of separation of the collided signals $Z_{L}(t)$ changes into that of the signals $Z(t)$. Here, we would recover collided tag signals from $Z(t)$. Firstly, we map the signals to an orthogonal/phase (IQ) plane and get a constellation. Fig. 2(a) shows an example of three collided tags. Then, we could find several clustering center. This problem is a typical clustering issue. If no training data are used, it will be through an unsupervised clustering method, such as $k$-means. Due to its higher complexity, we will not consider it here. Another method is to use a prior known prefix signal as training data to reduce the complexity. However, the drift of the prefix signal will result in poor clustering performance. In this sub-section, we will adopt an unsupervised clustering method via a histogram with lower computational complexity.

Let a mapped IQ plane paved by a number of grids, shown in Fig. 2(b). Then, we count the numbers of collision signal samples falling into grids. Hence, a 3D histogram w.r.t the counted numbers could be drawn in Fig. 2(c). Next, the work is only to find peak columns, which will correspond to the centers of clusters. To reduce computational complexity, further, we could search the peaks in $2 \mathrm{D}$ histogram shown in Fig. 2(d), which is an I or Q-plane projection of Fig. 2(C). In fact, the centers of clusters could be expressed as functions w.r.t tag's channel coefficients. For example, when the number of the collided tags is three, the received signal becomes

$$
Z(t)=h_{0} c_{0}(t)+h_{1} c_{1}(t)+h_{2} c_{2}(t)+n_{0}(t)
$$

In this example, there are eight clustering centers, $h_{0}+h_{1}+h_{2}, h_{0}+h_{1}-h_{2}, h_{0}-h_{1}+h_{2},-h_{0}+h_{1}+h_{2}$, $h_{0}-h_{1}-h_{2},-h_{0}-h_{1}+h_{2},-h_{0}+h_{1}-h_{2}$ and $-h_{0}-h_{1}-h_{2}$. Through calculating Euclidean distances between each sample point of $Z(t)$ and the clustering centers, respectively, we would make a decision for each tag signal and separate it from the collided tag signals.

\section{SimUlation RESUlTS}

We evaluate the performance of the proposed tag collision resolution by computer simulations. In the simulations, we consider a scenario with a single reader and some passive tags. Once the tags enter the reader's zone, they will not leave until all the tags are successfully identified. When mu ltiple tags select a slot simu ltaneously, the tag signals will collide with each other. Then we will separate the collided signals on the PHY layer and decode the tag signals. We individually perform each simulation 5000 times, and average 5000 simulation results into the final results. 


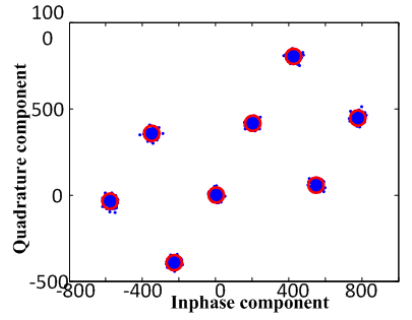

(a) A Constellation mapped from a collision signa to an IQ plane.
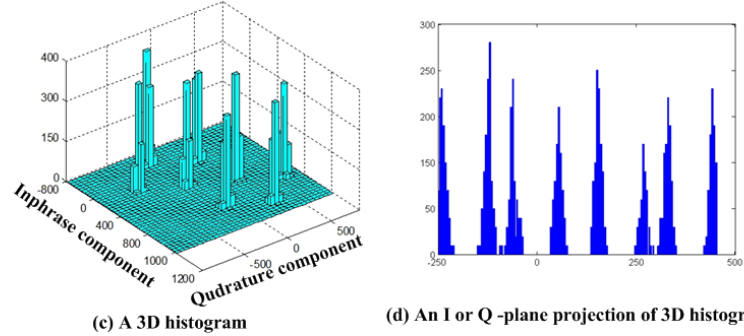

(d) An I or Q -plane projection of 3D histogram

FIGURE II. A DIAGRAM FOR UNSUPERVISED SEPERATION OF THREE COLLIDED T AG WHEN SNR IS 20DB

Some system parameters in this simulation are referenced to EPC C1 Gen2 standard [15], and the other ones are referenced to literatures [5, 6 and 8]. The detailed parameters are as follows.

- Channel: the channels are linear time-invariant (LTI) and flat fading during one identification cycle. And, each tag participating the collision experiences the same path loss. Thus we let $E\left\{\left|h_{j}\right|^{2}\right\}=1[6]$ which implies that no capture effect would happen

- Code: FM0 with $M=1[5,15]$, where $M$ is a symbol period multiplier.

- Decode: Viterbi algorithm [5]

- Nominal link frequency: $f_{l p}=44.4 \mathrm{kHz}$ [15]

- Symbol rate: each tag's symbol rate deviates up to $\pm 22 \%$ from the nominal link frequency, and the symbol rate deviation among tags is also up to $\pm 22 \%$ [5, 6 and 15]. Hence, we let $T_{j}$ produced in $\left[0.78 f_{l p}, 1.22 f_{l p}\right]$, and $\left|T_{i}-T_{j}\right| / T_{j}<22 \%$.

- Delay: $\tau_{j}<24 u s[5,6,15]$

- $\quad$ Sampling frequency: $32 \mathrm{MHz}$ [8]

- Dynamic scale: $b_{i}$ is randomly produced from $\left[\min \left(T_{i}\right), \max \left(T_{i}\right)\right]$

TABle I. The Probabiliry that $q$ Tag Signals Can Be Successfully RECOVERED FROM $r$ T AG Signals.

\begin{tabular}{|l|l|l|l|l|l|}
\hline & $\mathbf{q}=\mathbf{1}$ & $\mathbf{q}=\mathbf{2}$ & $\mathbf{q = 3}$ & $\mathbf{q}=\mathbf{4}$ & $\mathbf{q = 5}$ \\
\hline $\mathbf{r}=\mathbf{2}$ & 0.13 & 0.83 & 0 & 0 & 0 \\
\hline $\mathbf{r}=\mathbf{3}$ & 0.20 & 0.40 & 0.20 & 0 & 0 \\
\hline $\mathbf{r}=\mathbf{4}$ & 0.28 & 0.25 & 0.10 & 0 & 0 \\
\hline $\mathbf{r}=\mathbf{5}$ & 0.30 & 0.15 & 0.05 & 0 & 0 \\
\hline
\end{tabular}

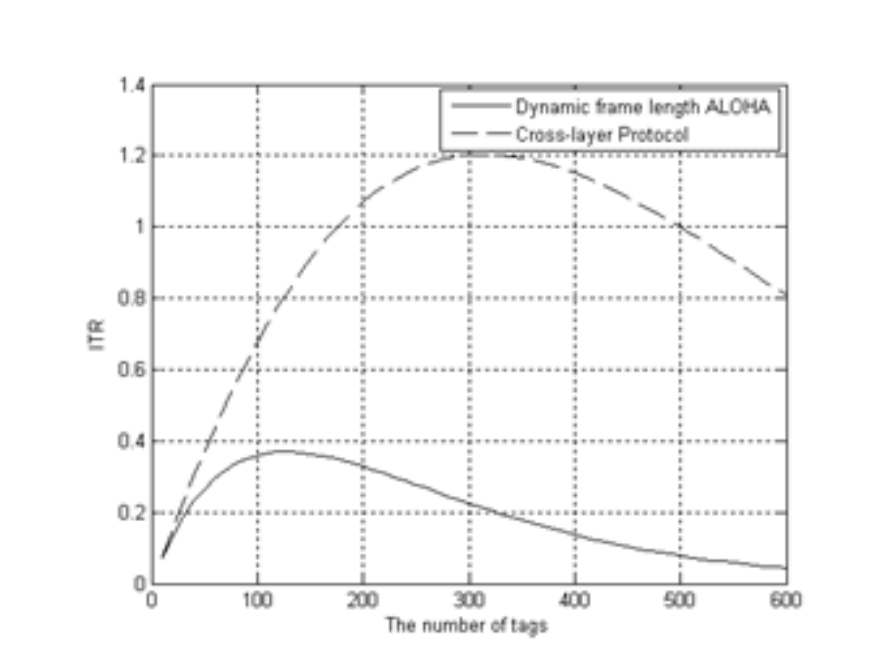

FIGURE III. ICR OF THE DYNAMIC FRAME LENGTH ALGORITHM AND THE CROSS-LAYER APPROACH

- $\quad$ Block length: The length is identical to that of RN16 specified in EPC C1 Gen2 [15], i.e. $K=32$

- Antenna:single receiving antenna

To evaluate the performance of the tag collision resolution, we consider the metric of identified tags to total slots ratio (ITR), where ITR is defined as

$$
I T R=n_{r} / n_{c} \times 100 \%
$$

in which $n_{r}$ and $n_{c}$ denote the number of successfully recovered tags and the number of collided slots, respectively. For the recovered tags, we regard them as successfully recovered ones only if there are no ID symbol errors. That is, the recovery will fail as long as there is a symbol error for the tag. Higher ITR will produce better performance of identification efficiency.

Table I gives the probability that $q$ tag can be successfully recovered from $r$ tags through the proposed resolution on PHY when signal to noise ratio (SNR) is $10 \mathrm{~dB}$. From the table, we can see that $q=0.04$ when $r=2, q=0.20$ when $r=3$, $q=0.37$ when $r=3$ and so on. The result indicates that more collided tags will produce higher recovered failure probability. That means that we make sure reasonable frame length for the number of tags on a MAC layer if higher recovered efficiency is guaranteed. Therefore, dynamic frame length ALOHA is a good choice because its expected number of tags in each collided slot is about 2.33[3].

Fig. 3 gives the ITR curves dynamic frame length ALOHA and the proposed cross-layer approach. In Fig. 3, the frame length $l=128$, and the number of tags $R$ varies from 10 to 100. From the figure, the maximum ITR of the ALOHA algorith $\mathrm{m}$ is only about 0.4 , whereas the maximu $\mathrm{m}$ ITR of the cross-layer approach arrives at 1.1. Furthermore, the ITR of the cross-layer approach is always higher than the ALOHA algorithm regardless of the number of tags. Therefore, the result shows that the proposed cross-layer approach has better 
performance of identification efficiency than the traditional dynamic frame length ALOHA.

\section{CONCLUSION}

This paper proposes an unsupervised separation algorithm for the passive UHF RFID tag collision resolution both on a MAC and PHY layer. From the simulation results, the proposed algorithm has better identification efficiency performance regardless of the number of tags. In addition, the number of tags in a collision slot will have an impact on the proposed recovery on a PHY layer. More collided tags will result in lower recovery efficiency. Thus, we should guarantee fewer tags in a collision slot and dynamic frame length ALOHA is a good candidate for the approach on a MAC layer.

Besides, it is admitted that our algorithm requires higher SNR although the unsupervised algorithm has lower complexity. Otherwise, an IQ constellation contaminated by noises will result in poor clusters, where peaks are difficult to find. However, an UHF RFID system is a near-field communication system, which could guarantee higher SNR.

\section{ACKNOWLEDGMENTS.}

This work was supported by the National Natural Science Foundation of China under Grant No. 61762093, The17th batches of Young and Middle-aged Leaders in Academic and Technical Reserved Talents Project of Yunnan Province under Grant No. 2014HB019, Supported by Program for Innovative Research Team (in Science and Technology) in University of Yunnan Province.

\section{REFERENCES}

[1] Finkenzeller, F. RFID Handbook: Fundamentals and Applications in Contact less Smart Cards, Radio Frequency Identification and Near-Field Communications, Third Edition. England: Wiley, 2010

[2] Liu, X., Kountouriotis, J., Petropulu, A.P., Dandekar, K. R.: ALOHA With Collision Resolution (ALOHA-CR): Theory and Soft ware Defined Radio Implementation. IEEE Transaction on Signal Processing, vol. 58, no. 8, pp. 4396-4410, 2010.

[3] Klair, D. K., Chin, K. W., Raad, R: A Survey and Tut orial of RFID Anti-Collision Protocols. IEEE Communicat ions Surveys \& Tut orial, vol. 12, no. 3, pp. 400 - 421, 2010.

[4] Zhu, F., Xiao, B., Liu, J.: Efficient Physical-layer Unknown Tag Identification in Large-scale RFID Systems. IEEE Trans on Commun, vol. 65 , no. 1, pp. 283-295, 2017.

[5] Fyhn, K., Jacobsen, R.M., Popovski, P., Scaglione, A., Larsen, T.: Multipacket Reception of Passive UHF RFID Tags: A Communication Theoretic Approach. IEEE Transactions on Signal Processing, Vol. 59, No. 9, pp. 4225-4237, 2011.

[6] Angerer, C., Langwieser, R., Rupp, M.: RFID reader receivers for physical layer collision recovery. IEEE Trans. Commun., vol. 58, no. 12, pp. 3526-3537, Dec. 2010.

[7] Benbaghdad, M., Fergani, B., Tedjini, S.: Towards a New PHY Layer Scheme for Decoding Tags Collision Signal in UHF RFID System. IEEE Commun Letters, vol. 20, no. 11, pp.2233-2236, 2016.

[8] Shen, D., Woo, G., Reed, D. P., Lippman, A. B., Wang, J.: Separation of multiple passive RFID signals using software defined radio. In Proc. IEEE Int. Conf. RFID, Orlando, FL(2009)

[9] Bletsas, A., Kimionis, J., Dimitriou, A. G., Karystinos, G. N.: Single-Antenna Coherent Detection of Collided FM0 RFID Signals. IEEE Trans. Commun., Vol. 60, No. 3, pp. 756-766, 2012
[10] Capetanakis, J.I.: Tree algorithms for packet broadcast channels. IEEE Trans. Information Theory, vol. 25, no. 5, pp. 505-515, 1979.

[11] Hush, D. R., Wood, C.: Analysis of tree algorithm for RFID arbitration. In Proc. IEEE International Symposium on Information Theory, pp. 107-107. IEEE Press, Cambridge: (1998)

[12] Information technology - Radio frequency identification (RFID) for item management-Part 6: Parameters for air interface communications at $860 \mathrm{MHz}$ to $960 \mathrm{MHz}$, International Standard ISO/IEC 18000-6, 2004.

[13] Vogt, H.: Efficient object identification with passive. RFID tags. In Proceedings of International Conference on Pervasive Computing, pp. 98-113. Springer-Verlag, Zurich (2002)

[14] Wu, H.F., Zeng, Y.: Bayesian Tag Estimate and Optimal Frame Length for Anti-Collision Aloha RFID System. IEEE Transactions on Automation Science and Engineering, vol. 7, no. 4, pp. 963-969, 2010.

[15] EPC radio-frequency identification protocols class-1 generation2 UHF RFID protocol for communications at $860 \mathrm{MHz}-960 \mathrm{MHz}$ Version 1.2.0 Draft 1, EPCglobal Inc, 2008

[16] Bonuccelli, M. A., Lonetti, F., Martelli, F.: Tree slotted ALOHA: a new protocol for tag identification in RFID networks. In Proceedings of International Symposium on a World of Wireless, Mobile and Multimedia Networks, pp. 1-6. IEEE Press, New York (2006)

[17] Wu, H.F., Zeng, Y., Feng, J., Gu, Y.: Binary Tree Slotted ALOHA for Passive RFID Tag Anti-Collision. IEEE Trans. Parallel and Distributed Sy stems, vol. pp, no. 99, pp. 1-14, 2012. 